\title{
Marius Köppel: ZEIT-FRAG-MENTE. Von Bedeutung auch für die Psychoanalyse (Eigenverlag, Zug, 2011)
}

\author{
Verena Schwerzmann (Zug)
}

Das Bewusstwerden von Zeit ist ein Themenkomplex, mit dem sich Marius Köppel schon in seinen früher erschienenen Schriften «Zerfall der Seele bei Descartes» 2011 und «Prähistorie von Zeit» 2012 auseinander gesetzt hat. Im vorliegenden Band zeichnet der Autor eine Entwicklungsgeschichte des menschlichen Zeiterlebens. Philosophische und psychoanalytische Erkenntnisse verknüpft er mit eigenen Denkansätzen, die sich zudem auf eine langjährige psychoanalytische Berufserfahrung abstützen.

Köppel geht in dieser Studie von der Annahme aus, dass unsere Ur-Vorfahren vor ungefähr sieben Millionen Jahren - erste Menschen bzw. Tiere, die langsam zu Menschen werden - noch ohne ein Bewusstsein von Zeit lebten. Es sind ungeheure Zeiträume, worin er die Genealogie unseres Zeitbewusstseins bzw. die «Prähistorie von Zeit» angesiedelt vermutet. Zeiträume, die durch zwei tiefgreifende Bruchstellen gekennzeichnet sind: Ein erster Umbruch erfolgte im Übergang von Leben in absoluter Gegenwart zu einem Leben, das von Vergänglichkeit weiss. Der zweite Umbruch bezieht sich auf die Erkenntnis von Zukunft und deren Folgen.

Der Autor versucht, den Wandel, die Umwälzungen, die mit diesen Entwicklungsschritten verbunden sind, darzustellen. Da das komplexe Thema nicht in der nötigen Ausführlichkeit bearbeitet werden kann, beschränkt sich Köppel darauf, eine Kernidee - jedoch mit wissenschaftlichem Anspruch - zu vermitteln. Es ist dies ein Unterfangen, das es dem Leser nicht ganz leicht macht, den Gedankengängen des Autors in einer «Zeitraffer-Methode» zu folgen.

Zeitbewusstsein in seinen Anfängen beschreibt Köppel als Leben in reiner Gegenwart (Wahrnehmungsgegenwart), ohne Vorstellung von Abwesenheit oder Kenntnis von Tod, genauso wie die uns bekannten Tierarten noch ohne Bewusstsein von Zeit über Jahrtausende hin allein in immerwährender Gegenwart existierten. «Nicht» kann nicht erfasst werden, weil in dieser frühen Zeit nur gesehen wird was ist.

Die Erkenntnis vom Tod muss daher eine riesige Umwälzung unter die Menschen gebracht haben, Einsicht in eine Tatsache, die wiederum Tausende 
von Jahren brauchte, um in ein selbstverständliches "Wissen» überzugehen. Damit ergab sich erst ein Bewusstsein von Vergänglichkeit bzw. Vergangenheit. Voraussetzung dafür war die Entwicklung der Fähigkeit, sich zu erinnern, d.h. über ein abrufbares Gedächtnis zu verfügen, nicht bloss auf Reize hin zu reagieren/assoziieren. Vermutlich liegt hierin - als Kompensation für den Verlust immer währender Gegenwart - die Wiege aller Religion. Von «Zukunft» hingegen ist noch keine Rede. Sie leitet dann die nächste Umstrukturierung ein: Dass wir ein spezifisches Wissen um Zukunft haben, Kenntnis von Dingen, die «noch nicht» sind, folglich nicht in Erscheinung treten, nicht wahrnehmbar sind, das ist eine sehr späte Errungenschaft. Eine ungefähre Vorahnung über ein «Zufliessen von Zeit» ist bei weitem noch kein klares Wissen um Zukunft, eher ein latentes, instinktives Spüren, dass es weitergeht (so wie die klebrigen Knospen am Kastanienbaum schon im Winter den nächsten Frühling erahnen lassen, während der Baum nichts davon weiss). Wissen um Zukunft hat sich gemäss Autor ergeben aus der Erkenntnis, wie Fortpflanzung funktioniert und sich in entsprechendem Handlungserfolg bestätigt, also vor etwa 9000 Jahren mit Heraufkommen und Nutzung von Ackerbau, Viehund Menschenzucht. Es war die Geburt der «Väter», Erfindung des «Patriarchats». Bis zu diesem Zeitpunkt gab es zwar Männer, aber keine Väter. Väter gibt es erst seit bekannt ist, wie Fortpflanzung durch Zeugung funktioniert.

Hier setzt der Autor auch mit Kritik an Freud ein: Einen «Vatermord» konnte es zu Beginn der Zeiten nicht geben, da es noch gar keine «Väter» gab. Sinnvollerweise kann ein Ödipuskomplex erst dann aufkommen, wenn es Väter gibt und diese als solche erkannt sind. Vorher gibt es auch keine solchen Verbände wie Familien. Die Zusammengehörigkeit war wohl eng zwischen den Müttern und ihren Sprösslingen; aber die Rolle der Männer war peripher, vielleicht auf Angeln und Jagen ausgerichtet, während die Frauen kreativ für den Nachwuchs sorgten, allenfalls noch Nahrungsmittel sammelten. Vermutlich lebte man in WirVerbänden. Freuds Aussagen waren diesbezüglich auch nie voll überzeugend, wurden bald angefochten und in Abrede gestellt. Neu und originell bei Köppel ist nicht die Anfechtung an sich, sondern die Art und Weise seiner Argumentation im Zusammenhang mit der Zeit-Genealogie: Weil Wissen um Zukunft mit dem Wissen um die Zeugungsvorgänge verknüpft ist, ist die Vaterfigur eine sehr späte Erscheinung. Wird der «Vater» an der Zeiten Anfang zurück transponiert, entspricht dies zwar häufigem Vorgehen, auf keinen Fall aber dem historischen, ja prähistorischen Prozess selber.

Das Mutterwerden der Frauen war immer offensichtlich, nur der Grund zur Schwangerschaft eben nicht. Der blieb rätselhaft. Man ist darum sehr geneigt, 
Mütterkulte als Ausdruck und Wunsch nach allgemeiner Fruchtbarkeit, somit auch die Muttergottheiten als um vieles ursprünglicher anzusehen als die erst späteren Götterfiguren/Vatergottheiten. Nach Köppel ist es in der Tat erstaunlich, wie widersprüchlich Freud sich in «Totem und Tabu» darüber äussert, obwohl er sieht und anerkennt, dass die Rolle des Mannes bei der Zeugung nicht auf Wahrnehmung, sondern auf einem «Schluss» beruhe, somit spätem abstraktem Denken entspringt.

War endlich der Mann als (möglicher) Vater erkannt, wurde die Frau als Mutter bildlich zu einem «Acker» herabgewürdigt. Jetzt waren Väter alles! Nachwuchs war Vaters Saat und die Frau nur die Matrix dafür - das Erdreich, welches die nötigeWärme, Feuchtigkeit und schonende Dunkelheit gewährt. Genetisch galt sie frei von jedem Einfluss; nur der Mann bot als Vater das Saatgut. Seither wurde die Frau dem Manne nach- und untergeordnet; dass sie als Mutter ihren ebenbürtigen genetischen Anteil leistet, ist erst seit knapp 200 Jahren bekannt, als man begann, sich für die Erbgesetze zu interessieren.

Mit der Entdeckung von Zukunft als offenem Terrain, das man als Erste besetzen will, sind nicht nur Besitztum und Herrschaft aufgekommen; es erforderte auch einen neuen (deduktiven) Typus von Religion - eine Religion, die über sinnliche Erfahrung hinausgeht und mit ihrem Konzept die aller Wahrnehmung sich entziehende Zukunft mit umfasst. Das war nur durch Offenbarung möglich, gestützt auf das verkündete Wort. Und der Gott dieser Religionen wurde mit sämtlichen Attributen ausgestattet, die menschlicher Errungenschaft entspringen, d.h. sie enteignen den Menschen seiner eigensten erfinderischen Taten, hängen sie ihrem Gott an und zwingen fortan die Gläubigen, sich diesem Gebilde zu unterwerfen. Darin sieht Köppel den Beginn einer Selbstentfremdung des Menschen von seiner begrenzten Natur, fortschreitend in ferne Zukunft.

Die moderne Fixierung aufZukunft wird durch die drei grossen monotheistischen Religionen unterstützt. Diese setzen ihr entscheidendes Ereignis an das Ende aller Zeiten. In säkularisierter Form äussert sich diese Fixierung auf Zukunft in einem extremen Befinden, das sich immer weiter von Vergangenheit und Gegenwart löst und sich einem Sog von Zukunftsblasen überlässt, die exzessiv ins Virtuelle führen. Gelebt wird nun mehr in fiktiven Welten. Wie kann da eine Psychoanalyse noch funktionieren, fragt Köppel, wenn das Interesse an Vergangenheit, an eigener Geschichte verblasst und die Menschen ihre eigene Innerlichkeit einbüssen? Bleibt nur das Verhalten, dessen momentane Äusserung, Bewertung, allenfalls die Korrektur nach Konventionen? Oder ist die alte Innerlichkeit, die noch persönlich war, nun an vorfabrizierte Programme abgetreten, die elektronisch z. B. in Form von Horrorgeschichten und/oder Pornostreifen usw. konsumiert werden? 
Der Autor äussert sich nicht näher dazu: Es kann auch sein, dass die Psychoanalyse nun erst recht zum Zuge kommt, war sie doch immer schon darauf angelegt, die «Realität» und deren Geschichten zu (re)konstruieren, und zwar aus Träumen, Fantasien, Wahn- und Wunschvorstellungen die «Realität» und deren Geschichten zu (re)konstruieren ... Aus der Masse heute anonym verfertigter und angeeigneter Virtualität könnte gerade sie helfen, eine persönliche Innerlichkeit trotz allem, ja die eigene Geschichte aus dem Wust von Bildern herauszufischen, sie erneut herzustellen, um wieder ein «Subjekt» zu errichten: Wo Es war, soll Ich werden! Besser noch: Ich und wir - denn ohne Wir (nicht: Über-Ich!) findet keiner sich zurecht.

Das Buch regt den Leser an, Köppels Leitidee - deren anschauliche, plausible Begründung noch recht provisorischer Natur ist - selber denkend zutage zu fördern.

\section{Literatur}

Köppel, M. (2011). Zerfall der Seele bei Descartes. Zug: Eigenverlag. Köppel, M. (2012). Prähistorie von Zeit. Zug: Eigenverlag. 